\title{
HOMOTOPY PERTURBATION METHOD FOR THE SOLUTION OF THE ELECTROSTATIC POTENTIAL DIFFERENTIAL EQUATION
}

LI-NA ZHANG AND JI-HUAN HE

Received 7 May 2006; Revised 5 July 2006; Accepted 16 July 2006

This paper obtains an explicit analytical solution for nonlinear Poisson-Boltzmann equation by the homotopy perturbation method, which does not require a small parameter in the equation under study, so it can be applied to both the weakly and strongly nonlinear problems. The obtained results show the evidence of the usefulness of the homotopy perturbation method for obtaining approximate analytical solutions for nonlinear equations.

Copyright (c) 2006 L.-N. Zhang and J.-H. He. This is an open access article distributed under the Creative Commons Attribution License, which permits unrestricted use, distribution, and reproduction in any medium, provided the original work is properly cited.

\section{Introduction}

Recently Oyanader and Arce [11] suggested a more effective, accurate, and mathematically friendly solution for prediction of the electrostatic potential, commonly used on electrokinetic research and its related applications. In order to obtain an explicit mathematical expression for the electrostatic potential, we have to solve the following nonlinear Poisson-Boltzmann equation:

$$
u^{\prime \prime}=\lambda^{2} \sinh u
$$

where $u$ is the dimensionless electrical potential, $\lambda$ the dimensionless inverse Debye length. The nonlinear term on the right-hand side of (1.1) is related to the free charge density. A very common simplification invokes the Debye-Hückel approximation usually written as

$$
\sinh u \approx u \text {. }
$$

As a result, the nonlinear Poisson-Boltzmann equation reduces to the following linear equation:

$$
u^{\prime \prime}=\lambda^{2} u
$$

Hindawi Publishing Corporation

Mathematical Problems in Engineering

Volume 2006, Article ID 83878, Pages 1-6

DOI 10.1155/MPE/2006/83878 
Such approximation is valid for the case when $-1 \leq u \leq+1$. In this paper, we will suggest an alternative approach to the search for an explicit analytical solution for (1.1) by homotopy perturbation method $[2,3]$.

\section{A brief introduction to homotopy perturbation method}

Homotopy perturbation method [2,3] is a novel and effective method, and can solve various nonlinear equations. Recently, some rather extraordinary virtues of the homotopy perturbation method have been exploited, and there has been a considerable deal of research in applying homotopy technique for solving various strongly nonlinear equations $[1,4-7,9,12,13]$, a complete review is available in [10], to illustrate its basic ideas of the new method, we consider the mathematical pendulum, the equation can be expressed as

$$
u^{\prime \prime}+\lambda^{2} \sin u=0
$$

with initial conditions $u(0)=A$ and $u^{\prime}(0)=0$.

Equation (2.1) can be approximated as follows:

$$
u^{\prime \prime}+\lambda^{2} u-\frac{1}{6} \lambda^{2} u^{3}=0, \quad u(0)=A, \quad u^{\prime}(0)=0
$$

According to the homotopy perturbation method, we construct a homotopy in the form

$$
u^{\prime \prime}+\omega^{2} u+p\left[\left(\lambda^{2}-\omega^{2}\right) u-\frac{1}{6} \lambda^{2} u^{3}\right]=0, \quad p=1
$$

where $p \in[0,1]$ is an embedding parameter, $\omega$ is the frequency of the mathematical pendulum which is to be determined further.

It is obvious that when $p=0,(2.3)$ becomes a linearized equation,

$$
u^{\prime \prime}+\omega^{2} u=0
$$

When $p=1$, it becomes the original nonlinear one. So the changing process of $p$ from zero to unity is just that of (2.4) to (2.2). The embedding parameter $p$ monotonically increases from zero to unity as the trivial problem (2.4) is continuously deformed to the studied problem (2.2). This is the basic idea of homotopy method which is to continuously deform a simple problem easy to solve into the difficult problem under study.

The homotopy perturbation method $[2,3,9]$ uses the imbedding parameter $p$ as a small parameter. The basic assumption is that the solution of (2.3) can be written as a power series in $p$ :

$$
u=u_{0}^{+} p u_{1}+p^{2} u_{2}+\cdots
$$

The convergence of the above series is discussed in [9], the asymptotic character of the series is illustrated in $[8,10]$. 
Substituting (2.5) into (2.3), collecting terms of the same power of $p$, gives

$$
\begin{gathered}
u_{0}^{\prime \prime}+\omega^{2} u_{0}=0, \quad u_{0}(0)=A, \quad u_{0}^{\prime}(0)=0, \\
u_{1}^{\prime \prime}+\omega^{2} u_{1}+\left(\lambda^{2}-\omega^{2}\right) u_{0}-\frac{1}{6} \lambda^{2} u_{0}^{3}=0, \quad u_{1}(0)=0, \quad u_{1}^{\prime}(0)=0 .
\end{gathered}
$$

Equation (2.6) can be solved easily, giving $u_{0}=A \cos \omega t$. If $u_{0}$ is substituted into (2.7), and the resulting equation is simplified, we obtain

$$
u_{1}^{\prime \prime}+\omega^{2} u_{1}+\left(\lambda^{2}-\omega^{2}-\frac{1}{8} \lambda^{2} A^{2}\right) A \cos \omega t-\frac{1}{24} \lambda^{2} A^{3} \cos 3 \omega t=0
$$

In order to avoid secular terms in $u_{1}$, $\omega$ is chosen to eliminate the coefficient of $\cos \omega t$. This condition determines $\omega$ to be

$$
\omega=\lambda \sqrt{1-\frac{1}{8} A^{2}} .
$$

The approximate period for mathematical pendulum reads

$$
T=\frac{2 \pi}{\lambda \sqrt{1-(1 / 8) A^{2}}}
$$

When $A=\pi / 2$, the value obtained from (2.10) is $T=1.20 T_{0}$, while the exact one is $T_{e x}=$ $1.16 T_{0}$, where $T_{0}=2 \pi / \omega$, the $3.4 \%$ accuracy is remarkably good in view of the crude approximation, $\sin x \approx x-(1 / 6) x^{3}$. If we approximate the sine function to the order $x^{5}$, $\sin x \approx x-(1 / 6) x^{3}+(1 / 120) x^{5}$, by the same manipulation as illustrated above, we obtain

$$
T=\frac{2 \pi}{\omega \sqrt{1-(1 / 8) A^{2}+(1 / 192) A^{4}+\cdots}} .
$$

The accuracy, for example, when $A=\pi / 2$, reaches $0.86 \%$.

\section{Explicit analytical solution for Poisson-Boltzmann equation}

We approximate the nonlinear Poisson-Boltzmann equation in the form

$$
u^{\prime \prime}=\lambda^{2}\left(u+\frac{1}{6} u^{3}\right)
$$

Suppose that the linearized equation of (1.1) can be expressed as

$$
u^{\prime \prime}=\omega^{2} u
$$

Hereby, $\omega$ is a modified inverse Debye length. Equation (3.2) is different from the DebyeHüchel approximation (1.3) in that the former is valid for the whole solution domain, while the latter for $-1 \leq u \leq+1$. 
Now we construct a homotopy in the form

$$
u^{\prime \prime}-\omega^{2} u-p\left[\left(\lambda^{2}-\omega^{2}\right) u+\frac{1}{6} u^{3}\right]=0
$$

As an illustrative example, we consider the following boundary conditions:

$$
u(-1)=A, \quad u(+1)=A, \quad u^{\prime}(0)=0 .
$$

Supposing that the solution of (3.3) can be expressed in the form of (2.5), by the same operation as illustrated in the above section, we obtain the differential equations for $u_{0}$ and $u_{1}$ :

$$
\begin{gathered}
u_{0}^{\prime \prime}-\omega^{2} u_{0}=0, \quad u_{0}(-1)=A, \quad u_{0}(+1)=A, \quad u_{0}^{\prime}(0)=0, \\
u_{1}^{\prime \prime}-\omega^{2} u_{1}-\left[\left(\lambda^{2}-\omega^{2}\right) u_{0}+\frac{1}{6} u_{0}^{3}\right]=0, \quad u_{1}(-1)=0, \quad u_{1}(+1)=0, \quad u_{1}^{\prime}(0)=0 .
\end{gathered}
$$

The solution of (3.5) is

$$
u_{0}=\frac{A \cosh \omega x}{\cosh \omega} .
$$

Substituting $u_{0}$ into (3.6) results in

$$
u_{1}^{\prime \prime}-\omega^{2} u_{1}-\left(\lambda^{2}-\omega^{2}\right) \frac{A \cosh \omega x}{\cosh \omega}-\frac{A^{3}(\cosh 3 \omega x+3 \cosh \omega x)}{24 \cosh ^{3} \omega}=0 .
$$

Collecting the cosh $\omega x$ term, and eliminating the cosh $\omega x$ term from (3.8) altogether, we have

$$
-\left(\lambda^{2}-\omega^{2}\right) \frac{A}{\cosh \omega}-\frac{A^{3}}{8 \cosh ^{3} \omega}=0
$$

or

$$
\omega^{2}=\lambda^{2}+\frac{A^{2}}{8 \cosh ^{2} \omega} .
$$

With this requirement, (3.8) becomes

$$
u_{1}^{\prime \prime}-\omega^{2} u_{1}-\frac{A^{3} \cosh 3 \omega x}{24 \cosh ^{3} \omega}=0 .
$$


The solution of (3.11), incorporating with the boundary conditions, $u_{1}(-1)=0, u_{1}(+1)=$ 0 , and $u_{1}^{\prime}(0)=0$, is

$$
u_{1}=\frac{A^{3}}{192 \omega^{2} \cosh ^{3} \omega}\left(\cosh 3 \omega x-\frac{\cosh 3 \omega}{\cosh \omega} \cosh \omega x\right) .
$$

So we obtain the first-order approximation, which reads as

$$
u=u_{0}+u_{1}=\frac{A \cosh \omega x}{\cosh \omega}+\frac{A^{3}}{192 \omega^{2} \cosh ^{3} \omega}\left(\cosh 3 \omega x-\frac{\cosh 3 \omega}{\cosh \omega} \cosh \omega x\right),
$$

where $\omega$ is solved from (3.10). In practice, we always use its zero-order approximate solution for simplicity:

$$
u=u_{0}=\frac{A \cosh \omega x}{\cosh \omega}
$$

\section{Conclusions}

In this paper, an explicit analytical solution is obtained for the nonlinear PoissonBoltzmann equation by means of the homotopy perturbation method, which is a powerful mathematical tool in dealing with nonlinear equations. The technique does require a small parameter in the equation under study. As a result, the technique eliminates completely the difficulty arising in the classic perturbation method.

\section{References}

[1] M. El-Shahed, Application of He's homotopy perturbation method to volterra's integro-differential equation, International Journal of Nonlinear Sciences and Numerical Simulation 6 (2005), no. 2, $163-168$.

[2] J.-H. He, Homotopy perturbation technique, Computer Methods in Applied Mechanics and Engineering 178 (1999), no. 3-4, 257-262.

[3] _ A coupling method of a homotopy technique and a perturbation technique for non-linear problems, International Journal of Non-Linear Mechanics 35 (2000), no. 1, 37-43.

[4] - Homotopy perturbation method: a new nonlinear analytical technique, Applied Mathematics and Computation 135 (2003), no. 1, 73-79.

[5] _ The homotopy perturbation method nonlinear oscillators with discontinuities, Applied Mathematics and Computation 151 (2004), no. 1, 287-292.

[6] _ Application of homotopy perturbation method to nonlinear wave equations, Chaos, Solitons \& Fractals 26 (2005), no. 3, 695-700.

[7] __ Homotopy perturbation method for bifurcation of nonlinear problems, International Journal of Nonlinear Sciences and Numerical Simulation 6 (2005), no. 2, 207-208.

[8] New interpretation of homotopy perturbation method, International Journal of Modern Physics B 20 (2006), no. 18, 1-7.

[9] _ Non-Perturbative Methods for Strongly Nonlinear Problems, Dissertation.de-Verlag im Internet GmbH, Berlin, 2006.

[10] Some asymptotic methods for strongly nonlinear equations, International Journal of Modern Physics B 20 (2006), no. 10, 1141-1199.

[11] M. Oyanader and P. Arce, A new and simpler approach for the solution of the electrostatic potential differential equation. Enhanced solution for planar, cylindrical and annular geometries, Journal of Colloid and Interface Science 284 (2005), no. 1, 315-322. 


\section{HPM for Poisson-Boltzmann equation}

[12] A. M. Siddiqui, M. Ahmed, and Q. K. Ghori, Couette and Poiseuille flows for non-newtonian fluids, International Journal of Nonlinear Sciences and Numerical Simulation 7 (2006), no. 1, $15-26$.

[13] A. M. Siddiqui, R. Mahmood, and Q. K. Ghori, Thin film flow of a third grade fluid on a moving belt by He's homotopy perturbation method, International Journal of Nonlinear Sciences and Numerical Simulation 7 (2006), no. 1, 7-14.

Li-Na Zhang: College of Science, Donghua University, Shanghai 200051, China E-mail address: livelyna@mail.dhu.edu.cn

Ji-Huan He: College of Science, Donghua University, Shanghai 200051, China E-mail address: jhhe@dhu.edu.cn 


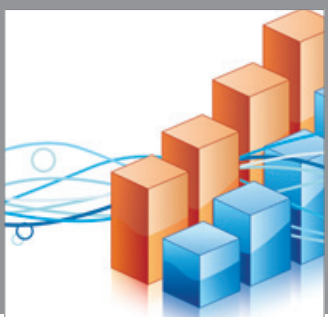

Advances in

Operations Research

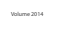

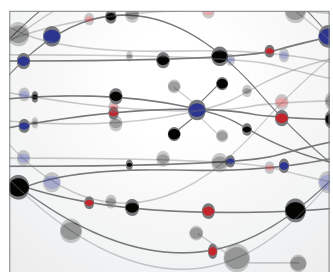

\section{The Scientific} World Journal
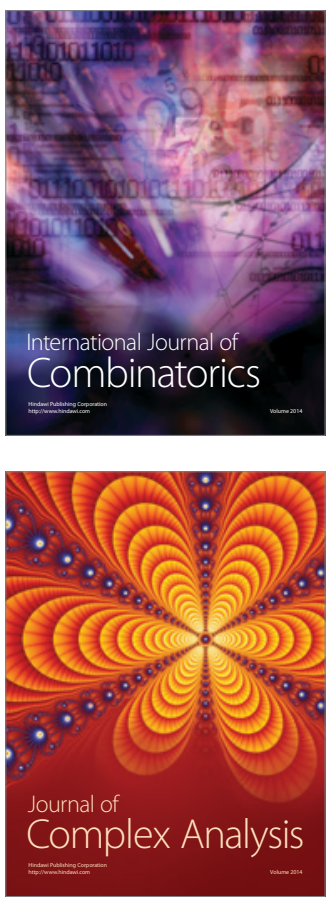

International Journal of

Mathematics and

Mathematical

Sciences
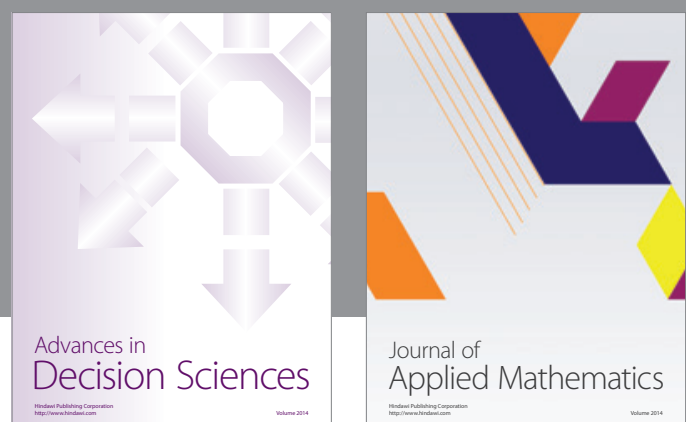

Journal of

Applied Mathematics
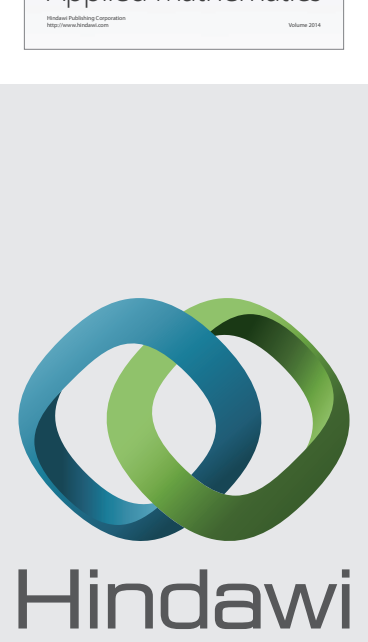

Submit your manuscripts at http://www.hindawi.com
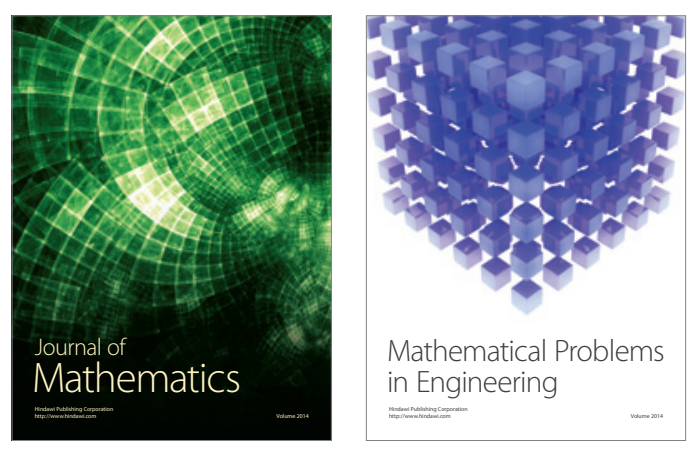

Mathematical Problems in Engineering
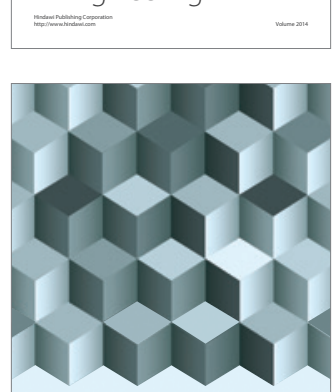

Journal of

Function Spaces
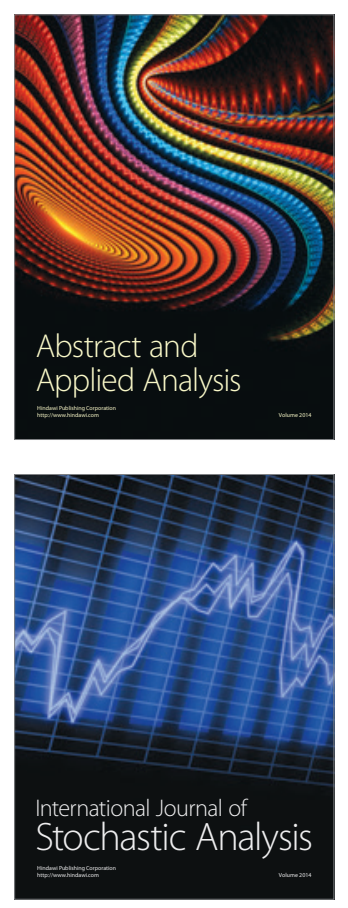

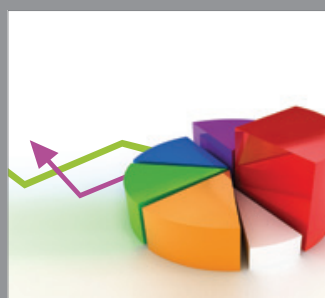

ournal of

Probability and Statistics

Promensencen
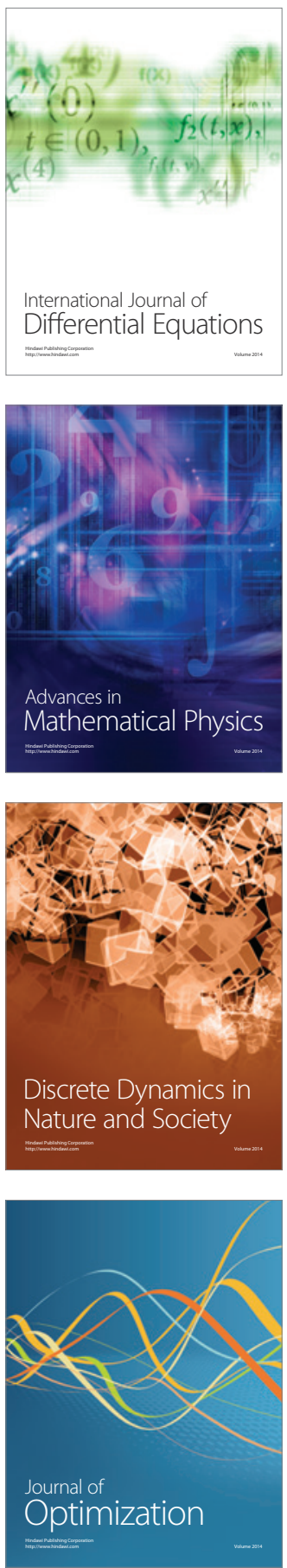